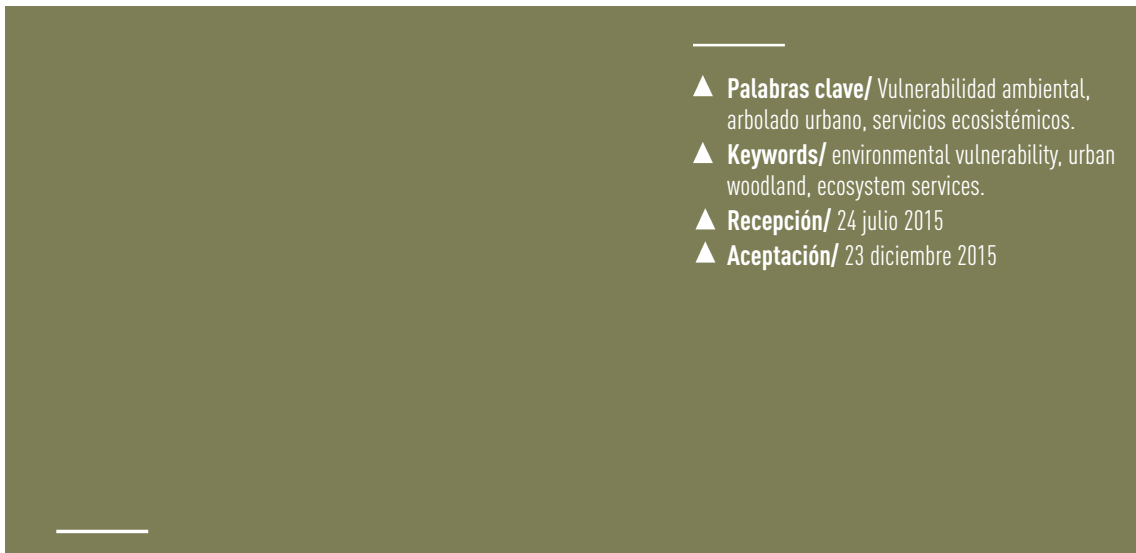

\title{
Vulnerabilidad ambiental del arbolado urbano. Levantamiento georreferenciado comunal, Chile ${ }^{1}$.
}

\section{Alexandre Carbonne}

Académico asistente, Escuela Arquitectura Universidad de Santiago, Chile.

Doctor Arquitecto Universidad Politécnica de Cataluña, España.

Arquitecto Universidad de Chile, Santiago, Chile.

alexandre.carbonnel@usach.cl

\section{Cecilia Aqueveque}

Estudio de Sustentabilidad Enjambre.

Geógrafa Universidad de Chile, Santiago, Chile. ceci.aqvq@gmail.com

\section{Mitzy Carmona}

Estudio de Sustentabilidad Enjambre.

Magíster (c) Teledetección, Universidad Mayor

Chile.

Geógrafa Universidad de Tarapacá, Chile.

mitzycarmonalopez@gmail.com

RESUMEN/ A partir de un catastro de árboles realizado durante los meses de junio a septiembre del año 2014, este estudio analiza la vulnerabilidad ambiental del arbolado urbano público (VAAUP) para

la comuna de Providencia, Chile. Las variables a considerar en el análisis fueron Fase de Crecimiento, Estado Fitosanitario y Estado de Afectación Biótica, los que combinados en una matriz de valoración muestran diferencias espaciales significativas de vulnerabilidad. Considerando la totalidad del área de estudio, las especies más representativas fueron Acer negundo L., Robina pseudoacacia L., y Platanus orientalis L. (el 50\% del total de la población arbórea). Las dos primeras especies son altamente vulnerables, lo que queda evidenciado en el mapa de VAAUP diseñado en un software de código abierto de Sistema de Información Goegráfica. Los resultados presentados buscan mejorar la gestión ambiental urbana de la comuna. ABSTRACT/ Based on a tree register made during June to September 2014, this study discusses the environmental vulnerability of public urban trees (EVPUT) in the district of Providencia, Santiago, Chile. The variables considered in the analysis are Growth Phase, Plant Health Status, and Biotic Stress Status, which combined in an assessment matrix show considerable spatial differences regarding vulnerability. Considering the entire study area, the most representative species were Acer negundo L., Robina pseudoacacia L., and Platanus orientalis L. (50\% of total tree population). The first two species are highly vulnerable, which is shown in the EVPUT map designed with a Geographic Information System’s open code software. The results aim at improving the commune's urban environmental management.

INTRODUCCIÓN. En Chile, la conservación

y administración de las áreas verdes, así como la propuesta, elaboración y ejecución de programas y normas ambientales, recae en los Departamentos de Aseo, Ornato y Medio Ambiente, según consta en la Ley N 18.695 Órgánica Constitucional de Municipalidades. Por ende, existe una relación directa entre la gestión ambiental, los presupuestos municipales y la calidad ambiental del arbolado.

Esta investigación estudia la vulnerabilidad ambiental del arbolado urbano público

(VAAUP) para la comuna de Providencia

U (ubicada en la Región Metropolitana), bajo el supuesto de que la actual composición de especies arbóreas y su estado fitosanitario no son los idóneos, acarreando altos costos de mantención y gestión para el municipio. En el año 2002, Providencia destinó un $71 \%$ del presupuesto del arbolado urbano público en la mantención de este, con un gasto de 1,69 dólares estadounidenses (USD) por árbol (Escobedo, Nowak, Wagner, De la Maza, Rodríguez, Crane y Hernández 2006), siendo junto a Vitacura (2,98 USD/árbol) una de las comunas con mayor gasto por árbol de la Región Metropolitana, cifras que contrastan con el gasto anual de comunas como La Pintana, Renca o Pudahuel, esta última con un gasto de solo 0,2 USD por árbol.
Con el objetivo de proporcionar una herramienta dinámica de gestión ambiental se georreferenciaron entre junio y septiembre del año 2014 un total de 41.720 árboles públicos (ubicados en calles, avenidas, paseos y pasajes, excluyendo áreas verdes y propiedades privadas). Esto permitió establecer microzonas prioritarias para el control de plagas, podas y unidades a reemplazar, mediante un mapa interactivo diseñado por el equipo de investigación con un software de código abierto (QGIS²). Entendemos que la mantención y distribución de la cobertura vegetal es muy relevante en cuanto a los servicios ambientales que presta. Un estudio 
de Romero, Opazo, Salgado y Smith (2010) por ejemplo, correlaciona la cobertura vegetal y sus efectos sobre la contaminación atmosférica según morfologías urbanas, sub-zonas climáticas y sectores socioeconómicos. Dicho estudio concluye que los sectores con menor cobertura vegetal e ingresos monetarios se ven expuestos a mayores tasas de enfermedades, principalmente respiratorias y cardiovasculares, bajo el mecanismo que los autores denominan "injusticia ambiental". En esta línea, y según nuestro sistema de organización administrativo territorial, donde los municipios son los administradores del territorio, el presente artículo propone una herramienta de valoración ambiental para mejorar la gestión y planificación ambiental comunal. En ese sentido, el mapa VAAUP facilita una visualización espacial que define priorización en el manejo ambiental leve y moderado, y en la remoción de árboles en estado irreversible, entregando una clasificación cualitativa y cuantitativa multivariable que facilita la toma de decisiones.

METODOLOGÍA. El análisis de VAAUP

se estructura sobre tres variables; fase de crecimiento, estado fitosanitario y tipo de afectación biótica. La combinación de estas variables permite parametrizar un mapa resultante de valorización a escala municipal (tabla 1)

\section{Variable A - Fase de crecimiento}

Según el estado en que se encontró cada especie, se categorizaron cuatro etapas o fases de crecimiento: Adulto, juvenil, sobremaduro y senescente. Se valoró que los árboles adultos presentan potencialmente mejores condiciones de

\section{resistencia al ambiente.}

\section{Variable B - Estado fitosanitario}

Basándose en la literatura existente (Alvarado, Bualdini y Guajardo 2013; Hoffmann 1998) y en la experiencia de los profesionales de terreno del estudio (ingenieros en recursos naturales, ingenieros forestales y geógrafos), se clasificó el estado fitosanitario en las siguientes categorías:
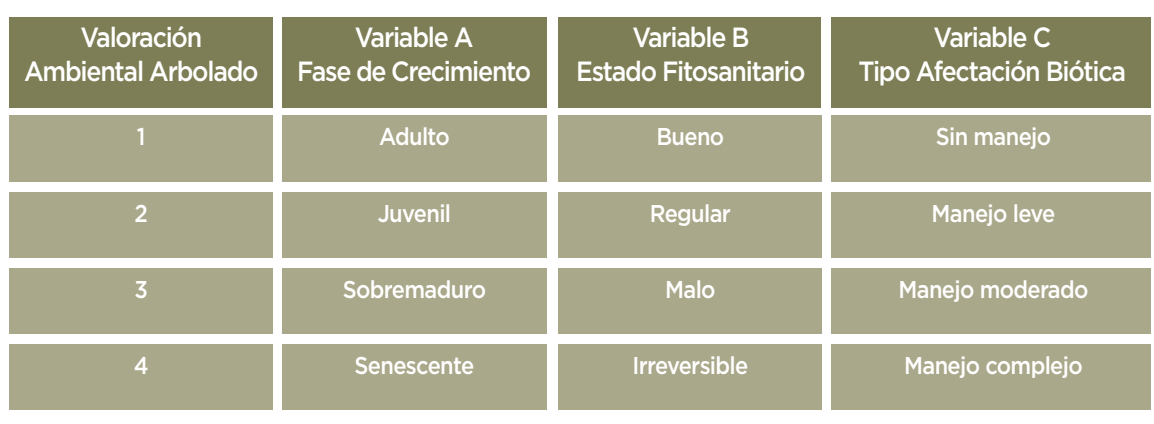

Tabla 1. Resumen sumatoria de valores para las tres categorías (fuente: Los autores 2014).
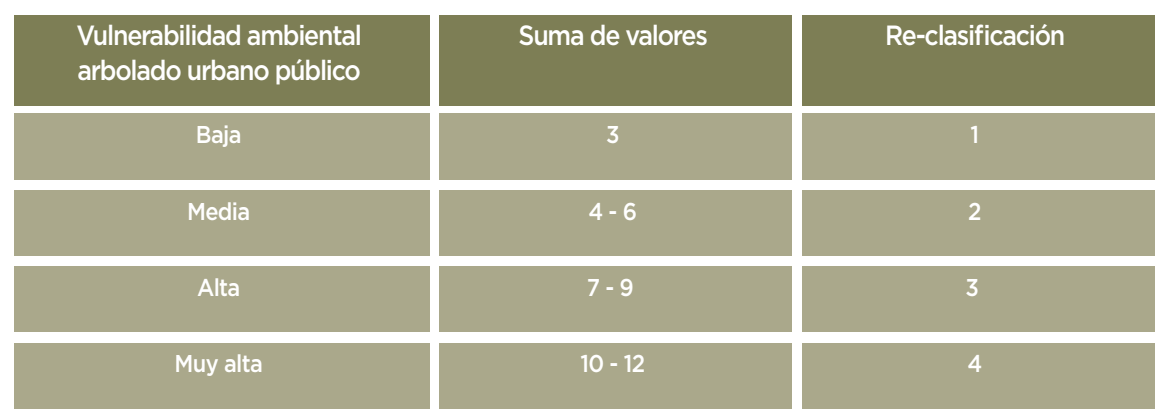

Tabla 2. Matriz de reclasificación VAAUP (fuente: Los autores 2014)

1. Bueno: Árbol de gran vigor, desarrollo normal de hojas, flores y frutos. Escasa o nula presencia de plagas o enfermedades. Buen anclaje, sin evidencia de debilidad. Muestra una estructura natural bien distribuida y estable.

2. Regular: Árbol en condición media de vitalidad. Problemas de sanidad, ya sea por presencia menor de insectos, plagas, enfermedades y/o daños mecánicos.

Recuperación posible a través de manejos.

3. Malo: Árbol de bajo vigor, posible pérdida del follaje, muerte apical o defoliación. Problemas serios de plagas o enfermedades como pudriciones, tumores, exudaciones y baja calidad de follaje. Con riesgo de estabilidad o sobrevivencia.

4. Irreversible: Árbol con daño evidente, mala o nula presencia de follaje, anclaje débil y malas condiciones en tronco y ramas. Afectado completamente por alguna plaga y/o enfermedad. Remplazo necesario.

\section{Variable C - Tipo de afectación biótica}

Se consideraron los principales agentes externos, tales como plagas, enfermedades, parásitos, hongos y epífitas. Entendiendo que las afectaciones conllevan una serie de recursos vinculados al manejo ambiental, se definió el siguiente puntaje de clasificación:

1. Sin afectación: Sin manejo

2. Afectación: Manejo leve

3. Enfermedad: Manejo moderado

4. Enfermedad + 1 ó más afectaciones:

Manejo complejo

Matriz de valoración ambiental del

arbolado. La sumatoria de puntajes arroja

el estado general en que se encuentra cada especie catastrada (tabla 2), siendo las de peor condición las que presentan vulnerabilidad Alta (7-9) o Muy Alta (10-12). Como vemos en la tabla 2, no existe aquí una priorización o ponderación según variables, ya que el objetivo es aportar información relevante y significativa, dejando un grado de flexibilidad en las decisiones de los técnicos que deberán ejecutar el plan de manejo ambiental del arbolado urbano público. 


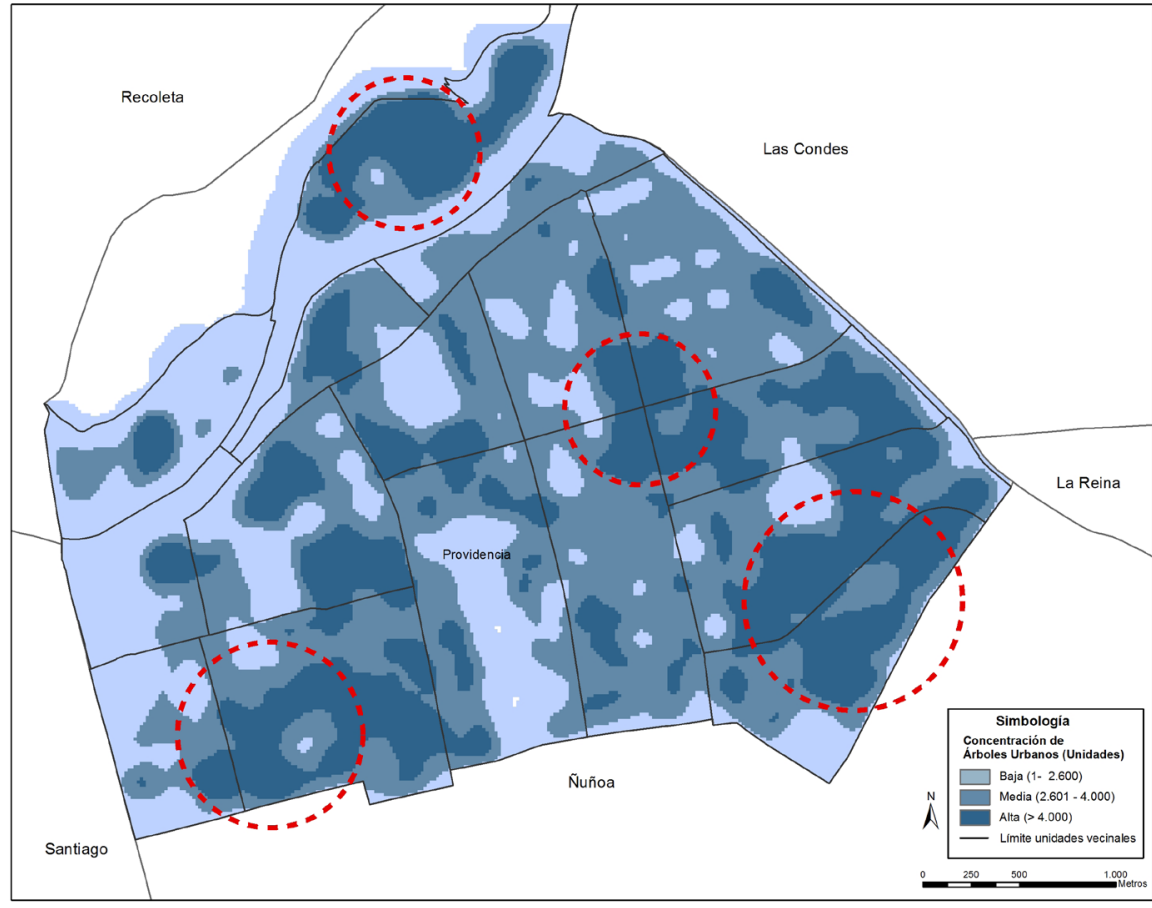

Imagen 1. Concentración de árboles (fuente: Los autores 2015).

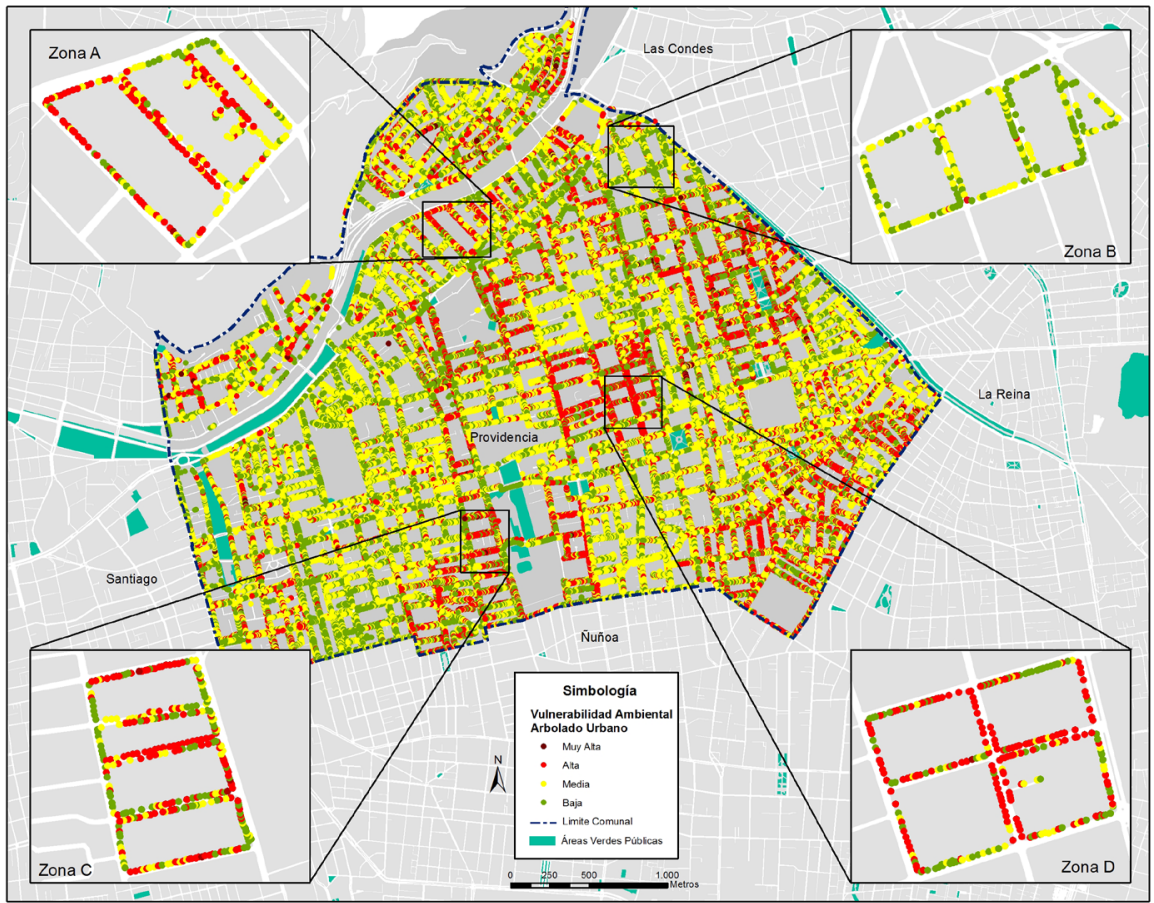

RESULTADOS. Las especies

más representativas del universo

georreferenciado fueron Acer negundo L.,

Robinia pseudoacacia L. y Platanus orientalis L., que en conjunto alcanzan las 20.717 unidades (49,65\% del total). A estas les siguen las especies prunus, liquidámbar, fresnos, celtis, melias, tulipero y jacarandá, en menores

\section{Vulnerabilidad ambiental}

En imagen 1 e imagen 2 se da cuenta de que la VAAUP no se correlaciona necesariamente con la densidad arbórea. Los resultados de la valoración multivariable muestran un espacio heterogéneo marcado por diferentes intensidades de VAAUP, observándose diferencias espaciales con cuatro microzonas representativas. La Zona B (imagen 2) aparece con una VAAUP baja, encontrándose árboles con un valor ponderado igual a 3 (tabla 3). Por ello, en este sector los árboles se encuentran, en su mayoría, en una fase de crecimiento adulta y no poseen indicios o presencia de plagas y/o enfermedades que debiliten su estructura.

Por el contrario, las otras tres zonas (A, C y D) manifiestan una alta VAAUP (imagen 2). Esto quiere decir que los mayores porcentajes de árboles que se presentan en la Zona A (43,45\%, 126 árboles), Zona C (42,67\%, 131 árboles) y Zona D (56\%, 168 árboles), poseen condiciones malas o débiles, ya sea porque tienen más de alguna afectación biótica (plagas o enfermedades) o porque se presentan en una temprana - muy avanzada etapa de crecimiento, lo que los vuelve más propensos a sufrir afectaciones. 


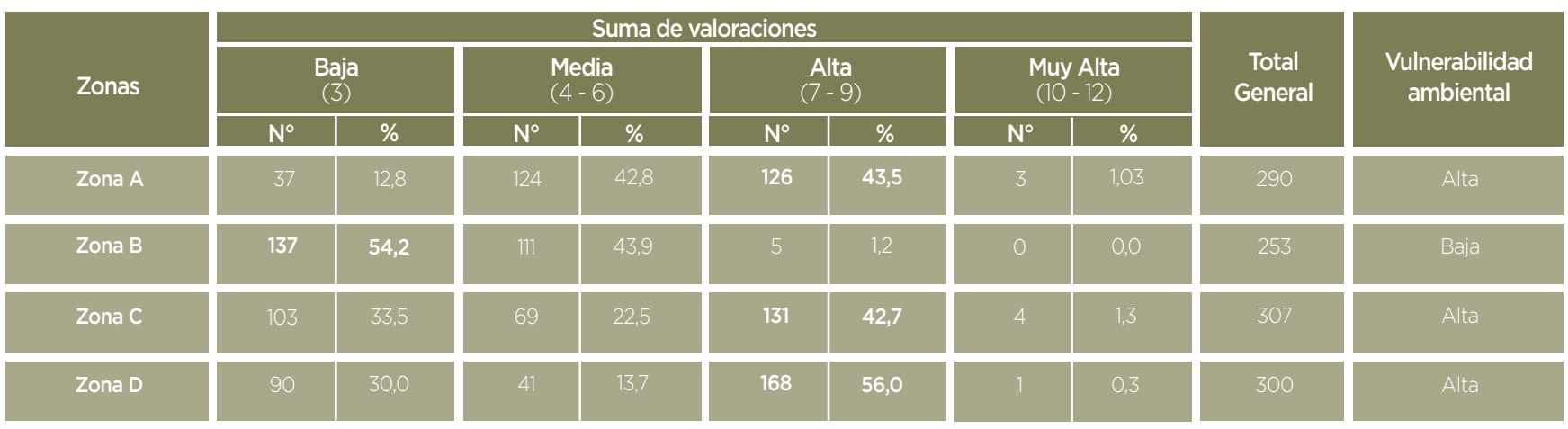

Tabla 3. Matriz de reclasificación VAAUP zonas A, B, C y D (fuente: los autores 2015).

\begin{tabular}{|c|c|c|c|c|c|c|c|c|c|c|}
\hline \multirow{3}{*}{$\begin{array}{l}\text { Nombre } \\
\text { Especie }\end{array}$} & \multirow{3}{*}{$\begin{array}{l}\text { Fase de } \\
\text { crecimiento }\end{array}$} & \multicolumn{9}{|c|}{ Estado Fitosanitario } \\
\hline & & \multicolumn{2}{|c|}{ Bueno } & \multicolumn{2}{|c|}{ Regular } & \multicolumn{2}{|c|}{ Malo } & \multicolumn{2}{|c|}{ Irreversible } & \multirow{2}{*}{ Total general } \\
\hline & & $\mathrm{N}^{\circ}$ & $\%$ & $\mathrm{~N}^{\circ}$ & $\%$ & $\mathrm{~N}^{\circ}$ & $\%$ & $\mathrm{~N}^{\circ}$ & $\%$ & \\
\hline $\begin{array}{l}\text { Acer } \\
\text { negundo } L \text {. }\end{array}$ & $\begin{array}{l}\text { Juvenil } \\
\text { Adulto } \\
\text { Sobremaduro } \\
\text { Senescente }\end{array}$ & $\begin{array}{c}317 \\
1353 \\
66 \\
0\end{array}$ & \begin{tabular}{|c|c|}
$77 \%$ \\
$35 \%$ \\
$5 \%$ \\
$0 \%$
\end{tabular} & $\begin{array}{c}79 \\
1730 \\
395 \\
8\end{array}$ & \begin{tabular}{l|l}
$19 \%$ \\
$44 \%$ \\
$29 \%$ \\
$15 \%$
\end{tabular} & $\begin{array}{c}15 \\
651 \\
504 \\
15\end{array}$ & \begin{tabular}{l|l}
$4 \%$ \\
$17 \%$ \\
$38 \%$ \\
$27 \%$
\end{tabular} & $\begin{array}{c}3 \\
154 \\
378 \\
32\end{array}$ & \begin{tabular}{r|}
$1 \%$ \\
$4 \%$ \\
$28 \%$ \\
$58 \%$
\end{tabular} & $\begin{array}{c}414 \\
3888 \\
1343 \\
55\end{array}$ \\
\hline Total & & 1736 & & 2212 & & 1185 & & 567 & & 5700 \\
\hline $\begin{array}{l}\text { Platanus } \\
\text { orientalis } L \text {. }\end{array}$ & $\begin{array}{l}\text { Juvenil } \\
\text { Adulto } \\
\text { Sobremaduro } \\
\text { Senescente }\end{array}$ & $\begin{array}{c}636 \\
2929 \\
424 \\
2\end{array}$ & \begin{tabular}{|c|c|}
$91 \%$ \\
$74 \%$ \\
$28 \%$ \\
$2 \%$
\end{tabular} & $\begin{array}{c}47 \\
894 \\
799 \\
112\end{array}$ & \begin{tabular}{|c|c|}
$7 \%$ \\
$22 \%$ \\
$52 \%$ \\
$88 \%$
\end{tabular} & $\begin{array}{c}8 \\
138 \\
257 \\
12\end{array}$ & \begin{tabular}{|l|l|}
$1 \%$ \\
$3 \%$ \\
$17 \%$ \\
$9 \%$
\end{tabular} & $\begin{array}{c}11 \\
24 \\
45 \\
2\end{array}$ & \begin{tabular}{|l|l|}
$2 \%$ \\
$1 \%$ \\
$3 \%$ \\
$2 \%$
\end{tabular} & $\begin{array}{c}702 \\
3985 \\
1525 \\
128\end{array}$ \\
\hline Total & & 3991 & & 1852 & & 415 & & 82 & & 6340 \\
\hline $\begin{array}{l}\text { Robinia } \\
\text { pseudoacacia } \\
\text { L. }\end{array}$ & $\begin{array}{l}\text { Juvenil } \\
\text { Adulto } \\
\text { Sobremaduro } \\
\text { Senescente }\end{array}$ & $\begin{array}{c}488 \\
2137 \\
197 \\
0\end{array}$ & \begin{tabular}{l|l|}
$85 \%$ \\
$47 \%$ \\
$6 \%$ \\
$0 \%$ \\
\end{tabular} & $\begin{array}{c}73 \\
1732 \\
1080 \\
18\end{array}$ & \begin{tabular}{|l|}
$13 \%$ \\
$38 \%$ \\
$32 \%$ \\
$24 \%$
\end{tabular} & $\begin{array}{c}9 \\
624 \\
1351 \\
23\end{array}$ & \begin{tabular}{l|}
$2 \%$ \\
$14 \%$ \\
$40 \%$ \\
$30 \%$
\end{tabular} & $\begin{array}{c}6 \\
89 \\
792 \\
35\end{array}$ & \begin{tabular}{|c|}
$1 \%$ \\
$2 \%$ \\
$23 \%$ \\
$46 \%$
\end{tabular} & $\begin{array}{c}576 \\
4582 \\
3420 \\
76\end{array}$ \\
\hline Total & & 2822 & & 2903 & & 2007 & & 922 & & 8654 \\
\hline Total general & & 8549 & 100 & 6967 & 100 & 3607 & 100 & 1571 & 100 & 20694 \\
\hline
\end{tabular}

Tabla 4. Asociación entre estado fitosanitario y fase crecimiento de especies prioritarias (fuente: Los autores 2015).

En los sectores centro y oriente de la comuna (imagen 2) se aprecian patrones sectoriales más extensos que evidencian VAAUP alta o muy alta. En estos lugares abundan árboles con afectación biótica en fase de crecimiento senescente, sobremaduro y adulto, detectándose estados fitosanitarios malos e incluso irreversibles, y observándose sectores con árboles con epífitas, enfermedades en sus hojas y plagas en sus frutos, tronco y ramas laterales.

En el sector centro-sur de la comuna existe una zona mixta de árboles con diversas calidades ambientales, mientras que en el sector sur-poniente abundan árboles con VAAUP baja o media. Aquí es común encontrar árboles como Liquidámbar (Liquidambar styraciflua L.) y Tulipero (Liriodendron tulipifera L.) en etapas juveniles y adultas con nula o baja presencia de plagas, parásitos o enfermedades, los que en muchos casos, con un manejo leve o moderado, se pueden recuperar rápidamente.

Como se aprecia en la tabla 4 y en los gráficos 1, 2 y 3, al analizar el estado fitosanitario y fase de crecimiento de las tres especies más representativas del arbolado urbano (Acer negundo L. (13,7\%), Robinia pseudoacacia L. (20,7\%) y Platanus orientalis L. $(15,2 \%)$ ), se destaca lo siguiente:

a) Las tres especies en etapa de crecimiento juvenil poseen características buenas y regulares. El Acer negundo L., con un $19 \%$, es la especie con más entidades regulares y el Platanus orientalis L., con un $91 \%$, es la especie con más especímenes buenos. El estado regular se manifiesta, principalmente, por daño mecánico asociado a factores externos y al incipiente desarrollo de enfermedades en sus hojas. 


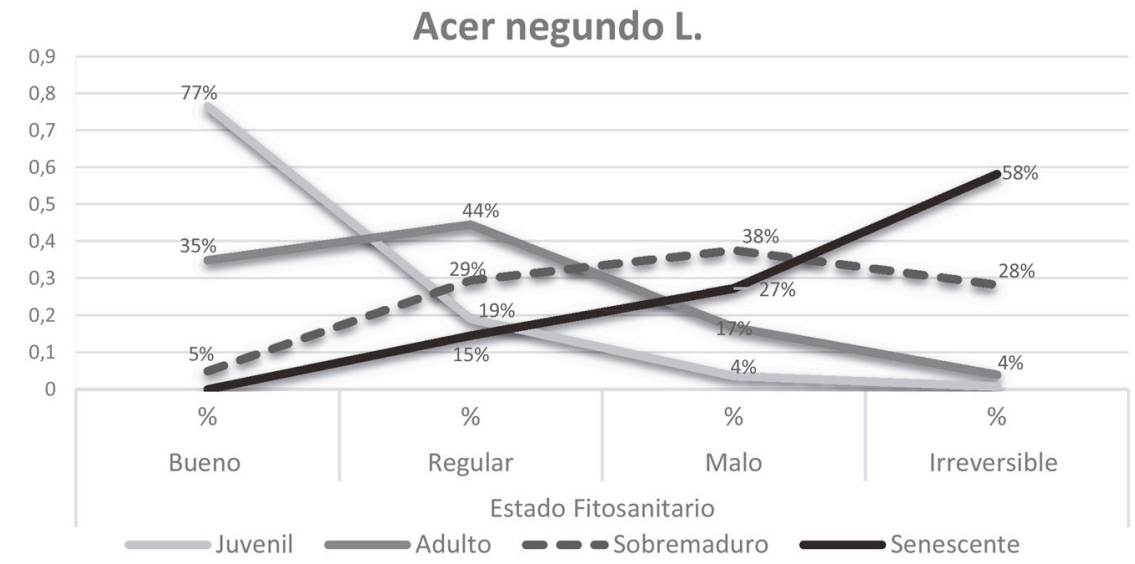

Gráfico 1. Porcentaje afectación estado fitosanitario Acer negundo (fuente: Los autores 2015).

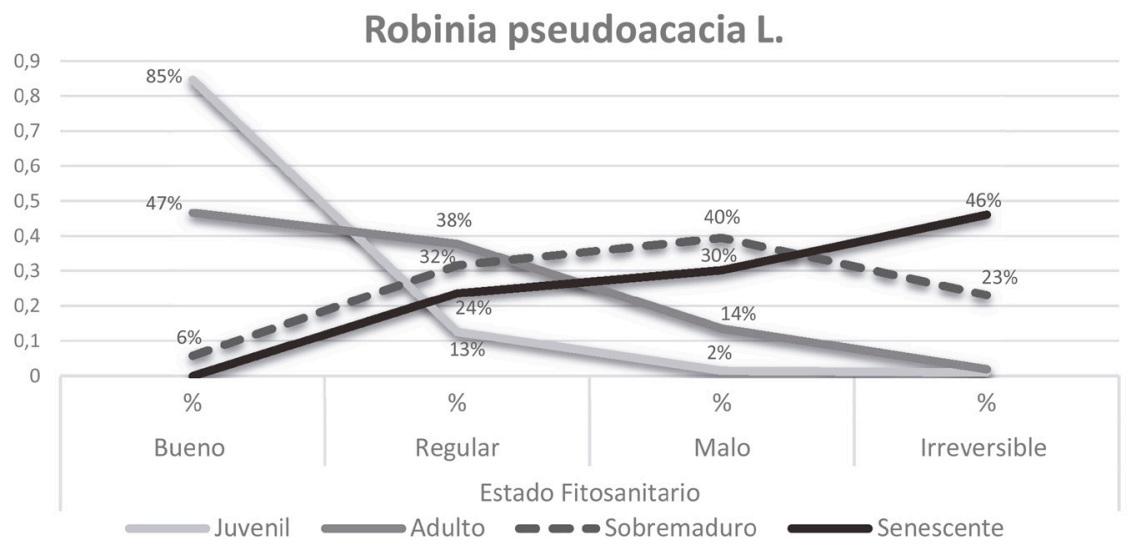

Gráfico 2. Porcentaje afectación estado fitosanitario Robinia pseudoacacia (fuente: Los autores 2015).

\section{Platanus orientalis L.}

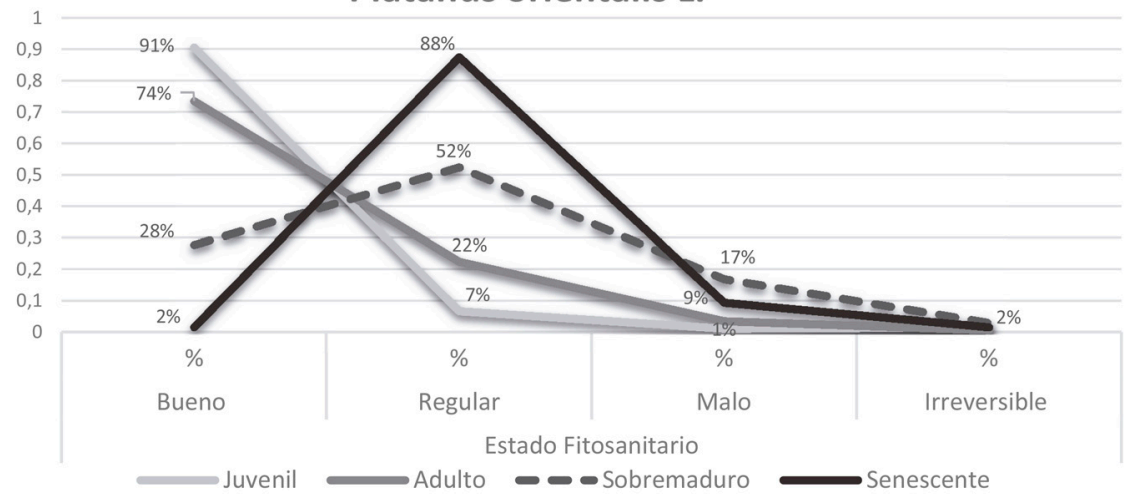

Gráfico 3. Porcentaje afectación estado fitosanitario Platanus orientalis (fuente: Los autores 2015). b) Los Arces y Robinias en una etapa adulta presentan mayoritariamente características regulares $(44 \%, 38 \%)$ y malas $(17 \%, 14 \%$ respectivamente), siendo sus principales afectaciones las plagas (taladradores) y enfermedades en tronco y hojas. En síntesis, los Arces (Acer negundo L.) en su etapa adulta, considerando el estado fitosanitario regular y malo, necesitan un manejo moderado y complejo para el 61\% de sus especímenes. En el caso de la Robinia pseudoacacia L., esto se da para un 52\%, y solo para un $25 \%$ en el caso de los Platanus orientalis $L$.

Estas dos especies, Arces y Robinias, presentan en su fase de crecimiento sobremaduro un estado fitosanitario irreversible de $28 \%$ y $23 \%$, respectivamente, lo que se ve acrecentado en su etapa senescente, con un $58 \%$ y $46 \%$,

presentándose la necesidad de reemplazar entidades debido a pudriciones avanzadas en troncos y ramas laterales.

c) El Plátano Oriental (Platanus orientalis L.) por su parte, no presenta afectaciones significativas en sus diferentes fases de crecimiento, concentrándose la mayor cantidad de entidades en estado malo (17\%) en su etapa de sobremaduro, lo que denota un estado fitosanitario, por lo general, bueno y regular. En su etapa adulta, la mayoría de sus entidades se encuentra en estado fitosanitario bueno (74\%) o regular (22\%). Lo mismo es evidente en su fase senescente, donde el 88\% de los árboles se encuentra en estado fitosanitario regular. 
con mal estado sanitario y sensible a las podas, ya que sus heridas son un conductor de hongos e insectos que propician plagas y enfermedades. El Arce, por su parte, es una especie de rápido crecimiento pero poco longevo y susceptible al ataque de hongos e insectos, principalmente en su fuste, por lo que su estado sanitario se va deteriorando rápidamente necesitando una mantención constante. El Plátano Oriental por el contrario, destaca por un rápido crecimiento, buen envejecimiento y resistencia a condiciones ambientales adversas, tales como heladas, contaminación, vientos fuertes y podas drásticas. Además, se caracteriza por mantener estados fitosanitarios buenos en su fase juvenil y adulta, siendo, dentro del arbolado urbano público analizado, una de las especies con menor vulnerabilidad. Se han evidenciado sectores donde especies exóticas han sido reemplazadas por nativas

\section{DISCUSIÓN.}

\section{Factores ambientales}

Los servicios ecosistémicos del arbolado urbano van desde la ornamentación y hábitat para la flora y fauna (corredores naturales), hasta la absorción de material particulado (especies perennifolias) atenuación de la contaminación acústica, regulación de temperaturas, Iluvia, viento y heladas, entre otros (Gutiérrez 2011: McPherson 2007)

En primera instancia, el estudio nos permite identificar sectores que poseen una VAAUP alta y muy alta, con condiciones precarias del arbolado. Por ende, es muy probable que estos árboles no produzcan efectos ambientales y servicios ecosistémicos óptimos, lo que será profundizado en investigaciones futuras a partir de los resultados expuestos en el presente artículo. De acuerdo a Alvarado, Baldini y Guajardo (2013), la Robinia es una especie longeva tales como Peumo y Quillay, las que según Gutiérrez (2006) son apropiadas para ser consideradas en ciudades, ya que toleran contaminación urbana, aunque no sucede lo mismo con aquella de origen industrial. Ambas especies pueden ser utilizadas en parques, plazas, jardines y vías de tránsito, incluso en bandejones centrales en su rango de distribución natural, desde la Región de Coquimbo hasta la Araucanía (Alvarado et al. 2013).

Según la imagen 2, pudiera existir una relación entre la ubicación espacial del arbolado y las condiciones ambientales que lo circundan, lo que afectaría de diferentes formas a una misma especie en iguales etapas de crecimiento. Sin embargo, para definir correlaciones directas, este mapa de VAAUP deberá combinarse con mapas térmicos y climáticos, análisis que será mostrado en futuros avances de la investigación.

\section{Factores urbanos: Espacio - Susceptibilidad}

A diferencia de otros espacios arbolados de la ciudad, una de las características que poseen los lugares estudiados (Díaz 2009) es que constituyen un conjunto de espacios longitudinales destinados al tránsito vehicular y peatonal, siendo su uso principalmente mixto. Existen, por ello, diversas situaciones que generan daños en el arbolado, como por ejemplo, ejes viales con alto tráfico, construcción de estacionamientos y remodelación de calles

\section{y vias públicas}

\section{Factores socioeconómicos}

En el área de estudio existe un total de 190 especies arbóreas, dónde solo tres concentran el $49,65 \%$ de la totalidad de árboles. Dos de ellas (Acer negundo L. y Robinia pseudoacacia L.) tienen mal envejecimiento y, por lo tanto, mayor VAAUP, lo que indica la necesidad de replantear los criterios de manejo y gestión del arbolado hacia otros factores ambientales y socio-urbanos.

Recientemente, se ha evidenciado una tendencia a la incorporación de nuevas especies endémicas de la zona central, reemplazándose a las especies prunus, robinias, arces y melias por otras del tipo perennifolias, tales como el Quillay (Quillaja saponaria) y Peumo (Cryptocarya alba) Este reemplazo disminuye los costos en mantención, ya que al ser especies endémicas de un clima mediterráneo, son más resistentes a la escasez de precipitaciones y al fuerte calor estival. Sin embargo, sólo el 8\% de especies fueron identificadas como nativas, universo muy bajo y poco significativo aún para establecer conclusiones sobre su impacto en la gestión municipal del arbolado urbano. 
CONCLUSIONES. El mapa de VAAUP permite mejorar el manejo del arbolado urbano, minimizando el gasto de intervención mediante tres actividades principales: plantación, mantención y reemplazo (Hernández, Bown, De la Maza y Raby 2004).

Según el estudio de Reyes (2014), el costo unitario de mantención de áreas verdes en la comuna de Providencia equivale a $301,9 \$ / \mathrm{m}^{2} /$ mes (50\% para personal, $5 \%$ vehículos, máquinas y equipos, $25 \%$ insumos y $15 \%$ agua de riego). Cabe destacar que este servicio es externalizado mediante contratistas, por lo cual, aunque estos datos se refieran a áreas verdes y no al arbolado urbano público, nos permiten visualizar el potencial del mapa de VAAUP para la optimización de recursos humanos y monetarios del municipio.

El mapa de VAAUP permite optimizar los contratos de servicios. Actualmente, la empresa contratista realiza mantenciones por valores mensuales según requerimientos del municipio (personal 50\% e insumos 25\%).
La herramienta desarrollada permite solicitar el reemplazo y manejo leve o moderado (plagas, enfermedades, podas, etc.) por microzonas y entidades arbóreas mejorando la eficiencia de la gestión.

Por otra parte, la herramienta entrega un diagnóstico cualitativo y cuantitivo sobre las especies con mayor y menor VAAUP en el entorno urbano del municipio, orientando así las decisiones sobre las entidades arbóreas y las especies a reemplazar. De esta forma, las especies Acer negundo L. y Robinia pseudoacacia L. deberían, en el caso de tener un estado fitosanitario irreversible o malo, ser reemplazadas idealmente por especies nativas idóneas al clima y entorno urbano u otras como el Liquidámbar (Liquidambar styraciflua L.) y Tulipero ( Liriodendron tulipifera L.), que en sus fases juveniles y adultas presentan muy pocas afectaciones sanitarias.

En cuanto al Platanus orientalis L., ya se mencionaron las ventajas relativas a su estado fitosanitario. Sin embargo, para evaluar sistémicamente el interés de priorizar su plantación, se necesita profundizar en estudios sobre externalidades ambientales negativas, como la polinización y las alergias que provoca sobre la población humana.

Por último, este estudio y mapa de VAAUP entrega una herramienta de sensibilización y sociabilización de fácil comprensión para los diferentes actores del municipio, tanto administrativos (Direccion de Obras Municipales, Dirección de Tránsito, Servicio de Panificación, etc.), como civiles (juntas de vecinos, organizaciones de base, asociaciones de comerciantes, etc.), a la hora de tomar decisiones sobre la gestión ambiental del arbolado urbano público.

\section{Abertura}

La posibilidad de cruzar el mapa de VAAUP con estudios focalizados sobre los servicios ecosistémicos del arbolado se presenta como un campo de estudio de amplio interés a escala municipal. En cuanto a la remoción de contaminantes, un estudio de Escobedo y Nowak (2009) señala la segregación espacial y socioeconómica en cuanto a la distribución del arbolado urbano y la concentración de $\mathrm{PM}^{10}{ }^{3}$. Las comunas de bajos ingresos de Santiago registran concentraciones de PM10 de 84,4 toneladas anuales para el periodo 2000-2001, superando en un $43 \%$ las concentraciones de comunas de altos ingresos (59,1 toneladas anuales de PM10). Según el mismo estudio, del aire contaminado (O3, PM10, $\mathrm{NO} 2, \mathrm{SO} 2, \mathrm{CO}$ ) removido por el arbolado, el $60 \%$ fue a través de los árboles, con promedios de remoción de $12,5 \mathrm{~g} / \mathrm{m}^{2}$ de cobertura, siendo el PM10 el más significativo con promedios de $7,4 \mathrm{~g} / \mathrm{m}^{2}$ (55\%-60\% de la remoción).

Si pudiéramos identificar la remoción según gases y PM10 dentro de la comuna y correlacionarla con la VAAUP, podríamos optimizar la gestión ambiental de esta mediante una cuantificación monetaria de sus externalidades y servicios ecosistémicos, a través de tasas de compensación o impuestos específicos. Cabe recordar, sobre esto último, que el ahorro generado en Santiago por la remoción de contaminación del aire equivaldría a US $\$ 44,8$ millones al año, por lo cual desagregar estos datos a nivel municipal permitiría ampliar una discusión sobre las políticas públicas enfocadas al arbolado urbano, los servicios ecosistémicos y la calidad de vida en zonas urbanas (Hernández 2008).

\section{REFERENCIAS}

Alvarado, A., Baldini, A. y Guajardo, F., 2013. Árboles Urbanos de Chile. Guía de reconocimiento (segunda edición). Santiago: Corporación Nacional Forestal (CONAF)

Diaz. J.M. 2009. Arbolado Urbano Viario. (Consultado el 16 de mayo 2015). Disponible en:

http://www.arbolado.cl/pdf/arbolado_urbano_viario.pdf

Escobedo, F., Nowak, D., 2009. "Spatial heterogeneity and air pollution removal by an urban forest." Landscape and Urban Planning, 90 (3-4), 102-110.

Escobedo, F., Nowak, D., Wagner, J., De la Maza, C., Rodriguez, M., Crane, D.E. y Hernández, J., 2006. "The socioeconomics and management of Santiago de Chile's public urban forests." Urban Forestry \& Urban Greening, 4 (3-4), 105-114.

을 Gutiérrez, R., 2011. "Valor del Arbolado Urbano: La experiencia de la Municipalidad de Santiago." Revista Mundo

U. Forestal, 21,4-6.

Gutiérrez, R., 2006. 84 árboles para las ciudades de Chile.. Santiago: Ediciones Universidad Mayor.

${ }^{3}$ Particulate matter o material particulado respirable.
Hernández, J., 2008. "La situación del arbolado urbano de Santiago." Revista de Urbanismo, 18. Disponible en: http://revistaurbanismo.uchile.cl/index.php/RU/article/view/272

Hernández. J., Bown. H., De la maza, C. L. y Raby. D., 2004. "La Necesidad de Inventariar el Arbolado Urbano: El Caso de la Comuna de La Reina de Santiago de Chile." (En Seminario Internacional: Funciones y Valores del Arbolado Urbano). Publicaciones Misceláneas, 5, 26-49.

Hoffmann, A. 1998. Elárbol urbano en Chile. Santiago: Ediciones Fundación Claudio Gay

McPherson, G., 2007. "Benefit-Based Tree Valuation." Journal Arboriculture \& Urban Foresty, 331, 1-11.

Reyes-Päcke, S., De La Barrera, F., Dobbis, C., Spotorno, A. \& Pavez, C. 2014. Costos de mantención de las áreas verdes urbanas en Chile. Informe Final. Seminario Chile Área Verde. Santiago: Centro de Políticas Públicas UC,

Facultad de Agronomía e Ingeniería Forestal y CEDEUS, Pontificia Universidad Católica de Chile

Romero, H., Opazo, D., Salgado M., Smith, P. 2010. "Climas urbanos y contaminación atmosférica en Santiago de Chile." EURE, 36 (109), 35-62 